\title{
DISTRIBUTIVELY GENERATED CENTRALIZER NEAR-RINGS
}

\author{
C. J. MAXSON AND $\dot{K}$. C. SMITH
}

\begin{abstract}
Let $G$ be a finite group. $Q$ a group of automorphisms of $G$ and $\mathcal{C}(\mathcal{Q} ; G)$ the centralizer near-ring determined by the pair $(Q, G)$. In this paper we investigate the structure of those centralizer near-rings $\mathcal{C}(Q ; G)$ which are distributively generated. Particular attention is given to the situation in which $G$ is a solvable group.
\end{abstract}

1. Introduction. Let $G$ be a group written additively with identity 0 , but not necessarily abelian and let $M_{0}(G)$ denote the near-ring of zero-preserving functions on $G$. For any near-ring $N$ contained in $M_{0}(G)$, we say $N$ is distributively generated (d.g.) if there exists a monoid $\mathcal{S}$ of endomorphisms of $G$ that additively generates $N$. Thus if $N$ is d.g. by $\delta$ then every function $f$ in $N$ has the form $f=\sum_{i=1}^{t} \varepsilon_{i} \sigma_{i}$, $\varepsilon_{i} \in\{-1,1\}, \sigma_{i} \in \mathcal{S}$. For any group $\mathbb{Q}$ of automorphisms of $G$, the set of functions $\mathcal{C}(\mathbb{Q} ; G)=\{f: G \rightarrow G \mid f(0)=0, f \alpha(x)=\alpha f(x), \alpha \in \mathbb{Q}, x \in G\}$ is a subnear-ring of $M_{0}(G)$ called the centralizer near-ring determined by $Q$ and $G$. It is the purpose of this paper to initiate a study of the characterization of those centralizer near-rings that are distributively generated.

In this paper all groups will be finite, all near-rings will be finite, zero-symmetric and have an identity element. As in [3], when $Q$ is a group of automorphisms of a group $G, \theta(v)=\{\alpha v \mid v \in G\}$ is the Q-orbit containing $v$ and $e_{v}$ is the map in $\mathcal{C}(Q ; G)$ which is the identity on $\theta(v)$ and zero off $\theta(v)$. Further, for $v \in G$, $\operatorname{stab}(v)=\{\beta \in \mathbb{Q} \mid \beta v=v\}$. For basic definitions and results concerning near-rings we refer the reader to the book by Pilz [5].

Fröhlich, [1], in 1958 determined the structure of the near-ring $N$ generated by all inner automorphisms of a finite, nonabelian simple group $H$. This near-ring is simple and in fact $N=M_{0}(H)$. Later, Laxton [2] generalized Fröhlich's result to the following: Let $N \subseteq M_{0}(G)$ be a finite, simple d.g. near-ring with identity which is not a ring, then $N=M_{0}(G)$ and $G$ is a finite, nonabelian invariantly simple group. (Recall that a subgroup $H$ of a group $G$ is fully invariant if $\sigma(H) \subseteq H$ for each endomorphism of $G$.) As an application of Laxton's result we let $N=\mathcal{C}(\mathbb{Q} ; G)$ be a simple d.g. centralizer near-ring which is not a ring. Thus $\mathcal{C}(\mathbb{Q} ; G)=M_{0}(G)$ so $\mathbb{Q}=\left\{\mathrm{l}_{G}\right\}$ and $G$ is a nonabelian, invariantly simple group. Thus, as we shall see the work in this paper initiates a study of the problem suggested by Fröhlich that one investigate d.g. near-rings of $M_{0}(G)$ where $G$ is not invariantly simple.

Received by the editors February 17, 1982.

1980 Mathematics Subject Classification. Primary 16A76; Secondary 16A44, 16A48.

Kel' words and phrases. Centralizer-near rings, distributively generated near-rings. 
The results here are also related to the recent work of the authors and M. R. Pettet [4]. There we considered the question “When is a centralizer near-ring a ring?". It was found that the only rings that occur as centralizer near-rings $\mathcal{C}(\mathbb{Q} ; G), \mathbb{Q}$ a group of automorphisms of $G$, are direct sums of fields. In the d.g. case we find a similar situation when $G$ is a solvable group. In this special case in which $\mathcal{C}(\mathbb{Q} ; G)$ is d.g. we find that $\mathcal{C}(\mathbb{Q} ; G)$ is either a field or a direct sum of fields. This situation is illustrated in the following example.

EXAMPLE 1. Let $G=S_{3}$ and $\mathbb{Q}=$ Aut $S_{3}\left(=\operatorname{Inn} S_{3}\right.$ ). Under the action of $\mathbb{Q}$ on $G$ we have $S_{3}=\{(1)\} \cup \theta_{1} \cup \theta_{2}$ where $\theta_{1}=\{(12),(23),(13)\}$ and $\theta_{2}=\{(123),(132)\}$. Further $\operatorname{stab}(12)=\{I, I(12)\}, \quad \operatorname{stab}(13)=\{I, I(13)\}, \quad \operatorname{stab}(23)=\{I, I(23)\}$ and $\operatorname{stab}(123)=\operatorname{stab}(132)=\{I, I(123), I(132)\}$ where $I(a)$ is the inner automorphism determined by $a$. Let $e_{i} \in \mathcal{C}(\mathbb{Q} ; G)$ denote the identity function on $\theta_{i}$ and zero off $\theta_{i}$, $i=1,2$. Then using the results of $[3]$ it is easily seen that

$$
\mathcal{C}(\mathbb{Q} ; G)=\left\{0,1, e_{1}, e_{2}, \alpha e_{2}, e_{1}+\alpha e_{2}\right\}
$$

where $\alpha(123)=(132)$. Thus $\bigodot(Q ; G)=S_{1} \oplus S_{2}, S_{1}=\left\{0, e_{1}\right\}, S_{2}=\left\{0, e_{2}, \alpha e_{2}\right\}$, and so $\mathcal{C}(Q ; G)$ is a direct sum of fields. We note that $\mathcal{C}(Q ; G)$ is distributively generated by $\delta=\{I\}$. Hence distributively generated centralizer near-rings do occur.

We conclude this section with a short summary of the remainder of the paper. In the next section we present some general results about d.g. centralizer near-rings. In the final section we specialize to the case in which $G$ is a solvable group and apply our results to completely determine when $\bigodot(Q ; G)$ is d.g., $G$ solvable.

2. Preliminary results. In this section we develop general results needed for our study of d.g. centralizer near-rings. As we indicated above, the setting is as follows: $G$ is a finite group, $Q$ a group of automorphisms of $G$ and $N$ is a subnear-ring of $\mathcal{C}(\mathscr{Q} ; G)$. For convenience we shall call $N$ a basic subnear-ring of $\mathcal{C}(\mathbb{Q} ; G)$ if $e_{v} \in N$ for every $Q$-orbit $\theta(v)$ of $G$.

Proposition 1. Let $N$ be a basic d.g. subnear-ring of $\mathcal{C}(\mathbb{Q} ; G)$. If $H$ is a fully invariant subgroup of $G$ then every $Q$-orbit of $G-H$ is a union of cosets of $H$ in $G$.

Proof. Let $\delta$ be the set of endomorphisms of $G$ which are the generators for $N$. If $f \in N$ then $f=\sum \varepsilon_{i} \Phi_{i}$ where $\varepsilon_{i}= \pm 1, \Phi_{i} \in \delta$. For $v \in G-H, h \in H$, we have

$$
f(v+h)=\sum \varepsilon_{i} \Phi_{i}(v+h)=\sum \varepsilon_{i}\left(\Phi_{i}(v)+\Phi_{i}(h)\right)=\sum \varepsilon_{i} \Phi_{i}(v)+h^{\prime}
$$

for some $h^{\prime} \in H$, using the normality of $H$ in $G$. Thus $f(v+h)=f(v)+h^{\prime}$.

Suppose $v, v+h$ belong to different orbits. Then, since $e_{v} \in N, 0=e_{v}(v+h)=$ $e_{v}(v)+h^{\prime}=v+h^{\prime}$ which would mean $v \in H$, a contradiction. So $v+h \in \theta(v)$ for every $h \in H$ and $\theta(v)$ is a union of cosets of $H$.

Proposition 2. Let $N$ be a basic d.g. subnear-ring of $\mathcal{C}(Q ; G)$. Suppose $H$ is a fully invariant subgroup of $G$ and let $\bar{Q}$ be the automorphism group of $H$ which is obtained by restricting the elements of $Q$ to $H$. If $\bar{N}$ is the subnear-ring of $\mathcal{Q}(\bar{Q} ; H)$ consisting of the elements of $N$ restricted to $H$, then $\bar{N}$ is basic and d.g. 
Proof. The map $\psi: N \rightarrow \bar{N}$ defined by $\psi(f)$ is the restriction of $f$ to $H$ and is a homomorphism of $N$ onto $\bar{N}$. Since $N$ is basic in $\mathcal{C}(\mathcal{Q} ; G), \bar{N}$ is basic in $\mathcal{P}(\overline{\mathcal{Q}} ; H)$. Since $N$ is d.g., so is $\bar{N}$.

Proposition 3. Let $N$ be a basic d.g. subnear-ring of $\mathcal{C}(\mathbb{Q} ; G)$. If $H \neq\{0\}$ is a fully invariant abelian subgroup of $G$, then $H^{*}$ is an orbit.

Proof. By Proposition 2, $\bar{N}$ is a basic d.g. subnear-ring of $\mathcal{C}(\overline{\mathbb{Q}} ; H)$. Since $H$ is abelian, the set $\operatorname{End}(H)$ of all endomorphisms of $H$ is a ring. Hence $\bar{N}$ is a ring of endomorphisms of $H$.

Assume $H^{*}$ has two distinct orbits $\theta(h), \theta(\tilde{h})$. If $h+\tilde{h} \notin \theta(h)$, then since $e_{h} \in \bar{N}$ we have $0=e_{h}(h+\tilde{h})=e_{h}(h)+e_{h}(\tilde{h})=h$, a contradiction. On the other hand, if $h+\tilde{h} \in \theta(h)$ then $h+\tilde{h}=e_{h}(h+\tilde{h})=h$, and $\tilde{h}=0$, again a contradiction. So $H^{*}$ is one orbit.

Proposition 4. Let $N$ be a basic d.g. subnear-ring of $\mathcal{C}(Q ; G)$. If $H$ is a fully invariant subgroup of $G$ such that $G / H$ is abelian, then $G-H$ is one orbit.

Proof. Since $G / H$ is abelian then for every $v, w \in G-H, v+w=w+v+h$ for some $h \in H$. Since $N$ is d.g. then as in the proof of Proposition $1, f(v+w)=$ $f(v)+f(w)+h^{\prime}$ for every $v, w \in G-H, f \in N$ and some $h^{\prime} \in H$.

Suppose $\theta(v), \theta(w)$ are distinct orbits in $G-H$. If $v+w \notin \theta(v)$ then $0=$ $e_{v}(v+w)=e_{v}(v)+e_{v}(w)+h^{\prime}=v+h^{\prime}$ and $v \in H$, a contradiction. A similar contradiction is reached if $v+w \in \theta(v)$. So $G-H$ is an orbit.

Proposition 5. Let $G$ be a nilpotent group. If $N$ is a basic d.g. subnear-ring of $\mathcal{E}(\mathcal{Q} ; G)$ then $N$ is a field, $G$ is an abelian p-group of exponent $p$ and $\mathbb{Q}$ acts transitively on $G^{*}$.

Proof. Every nilpotent group contains a fully invariant subgroup $H \neq\{0\}$ such that $H$ is a subgroup of the center of $G$. Since $N$ is d.g. then $f(v+h)=f(v)+f(h)$ for all $f \in N, v \in G-H, h \in H$. By Proposition $1, v+h \in \theta(v)$. We have $v+h$ $=e_{v}(v+h)=e_{v}(v)+e_{v}(h)=v+0=v$ and $h=0$. This gives a contradiction unless $G=H$, hence $G$ is abelian and $N$ is a ring. By Proposition 3, $G^{*}$ is an orbit, so $G$ is an abelian p-group of exponent $p$ and $e(Q ; G)$ is a near-field. As a subring of $\mathcal{C}(\mathbb{Q} ; G), N$ must be a field.

3. Distributively generated $\mathcal{C}(\mathbb{Q} ; G), G$ solvable. Throughout this section $G$ is assumed to be a finite solvable group. It is our goal to show that if $\mathcal{C}(\mathbb{Q} ; G)$ is distributively generated with $G$ solvable then $\bigodot(\mathscr{Q} ; G)$ is a ring and thus a direct sum of fields (see [4]).

Since $G$ is solvable, its series of higher commutator subgroups $G=G^{(0)} \supset G^{(1)} \supset$ $G^{(2)} \supset \cdots \supset G^{(n)}=\{0\}$, where as usual $G^{(i)}=\left[G^{(i-1)}, G^{(i-1)}\right], i=1,2, \ldots, n$, forms a normal series whose factor groups are abelian. For our purposes it is important to observe that each higher commutator subgroup $G^{(i)}$ is a fully invariant subgroup of $G$. If $G$ is a solvable group such that $G^{(n)}=\{0\}, G^{(n-1)} \neq\{0\}$ then we shall say $G$ has derived length $n$. 
If $G$ has derived length 1 then $G$ is abelian and $\mathcal{Q}(\mathcal{Q} ; G)$ d.g. implies $\mathcal{Q}(\mathbb{Q} ; G)$ is a field by Proposition 3. The length 2 case is handled in the following theorem, and is illustrated by the example in $\S 1$.

THEOREM 1. Suppose $G$ is a solvable group with derived length 2 and $巳(Q ; G)$ is d.g. for some automorphism group $\mathcal{Q}$ of $G$. Then $\mathcal{E}(\mathcal{Q} ; G)$ is a ring and $G$ is a Frobenius group of order $q p^{n}, p$ and $q$ distinct primes.

Proof. As a consequence of Proposition 5 we may assume $G$ is not a nilpotent group, and hence not a $p$-group. Let $H=G^{(1)}$, a fully invariant nonzero subgroup of $G$. Since $G$ has length $2, H$ is abelian and $G / H$ is abelian. By Propositions 3 and 4 , $G^{*}$ has two orbits under $\mathcal{Q}^{*}$, namely $G-H, H^{*}$. It is known [4, Theorem 4] that since $G$ is not a $p$-group, $G$ must be a Frobenius group of order $q p^{n}$ with $p, q$ distinct primes. Moreover $H$ is the Frobenius kernel of $G$ and $H$ is an elementary abelian group with order $p^{n}$. A Frobenius complement for $G$ is a cyclic group $Q$ of order $q$. From a result of $\mathrm{S}$. Garrison (see the following lemma) we find that if $v \in G-H$ and $h \in H^{*}$ then $\operatorname{stab}(v) \nsubseteq \operatorname{stab}(h)$. Since $\mathcal{e}\left(\mathcal{U}^{\prime} ; G\right)$ is distributively generated, $H$ is $\mathcal{C}^{\prime}\left(G^{\prime} ; G\right)$-invariant and $\operatorname{stab}(v) \nsupseteq \operatorname{stab}(h)$. So there are no proper stabilizer containments among elements of $G^{*}$, and by [3, Theorem 4$], \mathcal{Q}(\mathcal{Q} ; G)$ is semisimple. Since there are two orbits in $G^{*}, \mathcal{Q}(\mathbb{Q} ; G)$ is a direct sum of two near-fields. Since $\mathcal{E}\left(\mathbb{Q}^{\prime} ; G\right)$ is d.g. it is a direct sum of two fields and hence a ring.

LEMMA (GARRISON). For distinct primes $p$ and $q$ let $G$ be a Frobenius group of order $q p^{n}$ having kernel $H,|H|=p^{n}$, and complement $Q,|Q|=q$. Suppose $\mathbb{Q}$ is a group of automorphisms of $G$ which acts transitively on both $H^{*}$ and $G-H$. If $v \in G-H$ and $h \in H^{*}$ then $\operatorname{stab}(v) \nsubseteq \operatorname{stab}(h)$.

Proof. Suppose $v \in G-H, h \in H^{*}$ with $\operatorname{stab}(v) \subseteq \operatorname{stab}(h)$. We have $|\theta(v)|=$ $(q-1) p^{n}=\left|\mathbb{Q}^{*}: \operatorname{stab}(v)\right|$. Let $\mathscr{B}=\{\alpha \in \mathbb{Q} \mid \alpha(n v+H)=n v+H$ for all $n, n=0$, $1, \ldots, q-1\}$ a subgroup of $\mathcal{Q}$. We note that $\operatorname{stab}(v) \subseteq \mathscr{B}, \mathscr{B}$ is a normal subgroup

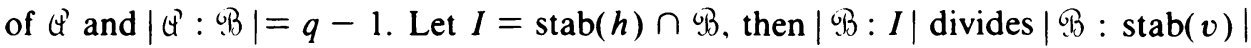

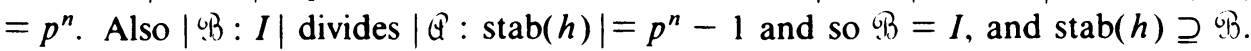
We have $q-1=|Q: G|=|\mathscr{Q}: \operatorname{stab}(h)||\operatorname{stab}(h): \mathscr{B}|=\left(p^{n}-1\right)|\operatorname{stab}(h): \mathscr{G}|$, and $|Q: \operatorname{stab}(h)|=p^{n}-1<q-1$. This is impossible since

$$
\begin{aligned}
(q-1) p^{n} & =\left|Q^{\prime}: \operatorname{stab}(v)\right|=\left|Q^{n}: \operatorname{stab}(h)\right||\operatorname{stab}(h): \operatorname{stab}(v)| \\
& =\left(p^{n}-1\right)|\operatorname{stab}(h): \operatorname{stab}(v)|
\end{aligned}
$$

and $q-1$ divides $p^{n}-1$.

Proposition 6. If $G$ is a solvable group with derived length 3 which is not a p-group and if $Q$ is any automorphism group of $G$, then $\mathcal{C}(Q ; G)$ is not distributively generated.

Proof. Assume $G$ is a solvable group with derived length 3 and with an automorphism group $Q$ such that $\mathcal{C}(\mathbb{Q} ; G)$ is distributively generated. Then $G \supset G^{(1)}$ $\supset G^{(2)} \supset G^{(3)}=\{0\}$. Let $H=G^{(1)}$ and $K=G^{(2)}$. From Propositions 2, 3,4 and Theorem 1, the orbits of $G^{*}$ are $G-H, H-K$ and $K^{*}$. Moreover if $\overline{\mathcal{Q}}$ is $\hat{\mathcal{Q}}$ restricted 
to $H$ then $\mathcal{C}(\overline{\mathbb{Q}} ; H)$ is distributively generated by Proposition 2 . From the proof of Theorem $1, H$ is a Frobenius group of order $q p^{n}$ with $[H: K]=q$ and $K$ is an elementary abelian group of order $p^{n}$. Since $\mathbb{Q}$ acts transitively on $G-H$ we must have $[G: H]=r^{m}$ for some prime $r$.

Assume $r \neq p, q$. Then $G-H$ contains an element of order $r$ and since $G-H$ is an orbit, then every element in $G-H$ has order $r$. Hence every element in $G^{*}$ would have order either $r, p$ or $q$. By Theorem 3 of [4], the only finite groups having the property that every nonidentity element has prime order are $p$-groups of exponent $p$, Frobenius groups of order $q p^{n}$, and the alternating group $A_{5}$. None of these is possible in this case. ( $A_{5}$ is excluded because it is not solvable.)

Assume $r=q$. Then $\tilde{G} \equiv G / K$ is a $q$-group. If $\tilde{Q}$ is the automorphism group on $\tilde{G}$ induced by $\mathscr{Q}$, then $\tilde{G}^{*}$ has two $\tilde{Q}$-orbits. The function $\Phi: \mathcal{C}(\mathbb{Q} ; G) \rightarrow \mathcal{C}(\tilde{\mathbb{Q}} ; \tilde{G})$ defined by $\Phi: f \rightarrow \tilde{f}$ where $\tilde{f}(a+G)=f(a)+G$, is a homomorphism of $\mathcal{C}(\mathbb{Q} ; G)$ into $\mathcal{C}(\tilde{\mathbb{Q}} ; \tilde{G})$. The image of $\Phi$ is a basic d.g. subnear-ring of $\mathcal{C}(\tilde{\mathbb{Q}} ; \tilde{G})$. By Proposition $5, \tilde{Q}$ acts transitively on $\tilde{G}^{*}$, a contradiction.

Assume $r=p$, which is the remaining possibility. The group $G / K$ has two nonzero orbits under the automorphism group induced by $Q$. By Theorem 4 of [4], the order of $G / K$ must be $p q$. This means $[G: H]=p$. We now have $|G|=q p^{n+1}$ with $[G: H]=p,[H: K]=q,|K|=p^{n}$ and $H$ is a Frobenius group of order $q p^{n}$. To prove no such group exists we use an argument suggested by $\mathrm{S}$. Gagola. Let $Q$ be a Sylow $q$-subgroup of $H$, a cyclic group of order $q$. By the Frattini argument [6,p. 88], $G=N_{G}(Q) H$. We have $\left|N_{G}(Q) \cap H\right||G|=\left|N_{G}(Q)\right||H|$, or

$$
\left|N_{G}(Q) \cap H\right| q p^{n+1}=\left|N_{G}(Q)\right| q p^{n}
$$

and so $p$ divides $\left|N_{G}(Q)\right|$. We claim $N_{G}(Q) \cap K=\{0\}$. Assume $v \in N_{G}(Q) \cap K$, $v \neq 0$. Then for $x \in Q, v+x-v=i x, 1 \leqslant i<q$. Hence $v+x-v-x \in Q \cap K$ $=\{0\}$. So $v+x=x+v$ which is impossible because $v+x$ would have order $p q$. So $N_{G}(Q) \cap K=\{0\}$ and $N_{G}(Q)$ contains an element of order $p$ not in $K$ and therefore in $G-H$. This means every element in $G-H$ has order $p$ and thus every element in $G^{*}$ has order $p$ or $q$, and $G$ is Frobenius by Theorem 3 of [4]. But such a Frobenius group would have a normal Sylow subgroup and our group does not, again a contradiction. Since none of the possibilities can occur we conclude that $\mathcal{Q}(\mathbb{Q} ; G)$ cannot be distributively generated.

Theorem 2. Suppose $\mathfrak{P}(\mathfrak{Q} ; G)$ is distributively generated and $G$ is solvable. Then $\mathcal{Q}(\mathbb{Q} ; G)$ is a ring and $G$ has derived length 2 .

Proof. From Theorem 1 and Proposition 6 it suffices to show that the derived length of $G$ cannot be larger than 3 . Assume $\mathcal{E}(\mathscr{G} ; G)$ is d.g. with $G$ having derived length 4. Let $H$ be the commutator subgroup of $G$ and let $\bar{Q}$ be the automorphism group on $\mathrm{H}$ induced by $Q$. Then $H$ has derived length 3 and $e(\bar{Q} ; H)$ is d.g., an impossible situation due to Proposition 6. Inductively it is seen that the derived length of $G$ cannot be larger. 


\section{REFERENCES}

1. A Fröhlich, The near-ring generated by the inner automorphisms of a finite simple group, J. London Math. Soc. 33 (1958), 95-107.

2. R. R. Laxton, Primitive distributively generated near-rings, Mathematika 8 (1961), 143-158.

3. C. J. Maxson and K. C. Smith, The centralizer of a set of group automorphisms, Comm. Algebra 8 (1980), 211-230.

4. C. J. Maxson, M. R. Pettet and K. C. Smith, On semisimple rings that are centralizer near-rings, Pacific J. Math. (to appear).

5. G. Pilz, Near-rings, North-Holland, New York, 1977.

6. J. J. Rotman, The theory of groups, an introduction, 2nd ed., Allyn \& Bacon, Boston, Mass., 1973.

Department of Mathematics, Texas A \& M University, College Station, Texas 77843-3368 